PROCEEDINGS OF THE

AMERICAN MATHEMATICAL SOCIETY

Volume 140, Number 1, January 2012, Pages 21-33

S 0002-9939(2011)10878-5

Article electronically published on May 6, 2011

\title{
PRODUCTS OF SQUARES IN FINITE SIMPLE GROUPS
}

\author{
MARTIN W. LIEBECK, E. A. O'BRIEN, ANER SHALEV, AND PHAM HUU TIEP
}

(Communicated by Jonathan I. Hall)

\begin{abstract}
The Ore conjecture, proved by the authors, states that every element of every finite non-abelian simple group is a commutator. In this paper we use similar methods to prove that every element of every finite simple group is a product of two squares. This can be viewed as a non-commutative analogue of Lagrange's four squares theorem. Results for higher powers are also obtained.
\end{abstract}

\section{INTRODUCTION}

In recent years there has been considerable interest in word maps on finite nonabelian simple groups $G$ : namely, maps of the form $\left(g_{1}, \ldots, g_{d}\right) \mapsto w\left(g_{1}, \ldots, g_{d}\right)$, where $w$ is a non-identity element of the free group $F_{d}$ of rank $d$ and $g_{i} \in G$. Let $w(G)$ denote the image of this map and let $w(G)^{k}$ be the set of all products of $k$ elements of $w(G)$. In [24] it is shown that $w(G)^{3}=G$ provided $|G|>N_{w}$, where $N_{w}$ depends only on $w$, and this has recently been improved to $w(G)^{2}=G$ in 16, 17, 18. Clearly there are words $w$ for which $w(G) \neq G$; for example $w=x^{2}$ is not surjective on any finite non-abelian simple group. More generally, any word which is a proper power is non-surjective on infinitely many simple groups: indeed, if $w=x^{k}$ and $|G|$ is not coprime to $k$, then the map $g \mapsto g^{k}$ is not injective on $G$, so $w(G) \neq G$. However, some word maps are surjective, and it is a major challenge to determine which ones are.

Trivially, any primitive word - that is, any word that is part of a free generating set of $F_{d}$ - is surjective on all groups. The same is true for any word of the form $\prod_{i=1}^{d} x_{i}^{e_{i}} f$ with $f \in F_{d}^{\prime}$, where $e_{1}, \ldots, e_{d}$ are integers with greatest common divisor 1 (see [23, 3.1.1]). The first non-trivial example of a word map which is surjective on all finite non-abelian simple groups is the commutator map $[x, y]$. Indeed, this is the content of the well known Ore conjecture that every element of a finite non-abelian simple group is a commutator, recently proved in [19. Its long proof combines

Received by the editors May 21, 2010 and, in revised form, November 1, 2010.

2010 Mathematics Subject Classification. Primary 20C33, 20 D06.

The first author acknowledges the support of a Maclaurin Fellowship from the New Zealand Institute of Mathematics and its Applications.

The second author acknowledges the support of the Marsden Fund of New Zealand (grant UOA 0721)

The third author acknowledges the support of ERC Advanced Grant 247034, an EPSRC Visiting Fellowship, an Israel Science Foundation Grant, and Bi-National Science Foundation grant United States-Israel 2008194.

The fourth author acknowledges the support of the NSF (grant DMS-0901241).

(C)2011 American Mathematical Society 
character theory and computational methods. In this paper we use these and other ideas to prove another surjectivity result, this time for the word $x^{2} y^{2}$.

Theorem 1. Every element of every finite non-abelian simple group $G$ is a product of two squares. In other words, if $w(x, y)$ is the word $x^{2} y^{2}$, then $w(G)=G$.

This proves a conjecture stated in [18. Note that by the general result of [18, every element of a sufficiently large finite simple group is a product of two squares; however, it is intriguing that no exception exists.

The word $x^{2} y^{2}$ is of interest for a number of reasons. Firstly, one can think of the theorem as a non-commutative analogue of Lagrange's four squares theorem. Secondly, it was shown in 8 that the word $x^{2} y^{2}$ is almost measure-preserving on finite simple groups - namely, the inverse image of a subset $S$ of $G$ of proportion $p=|S| /|G|$ has proportion $p+o(1)$ in $G \times G$ as $|G| \rightarrow \infty$ - but its surjectivity remained open. Thirdly, as for commutators, there is a character theoretic connection essentially going back to Hurwitz (see Lemma 2.2). This paves the way to character theoretic methods which are used in our proof of the theorem.

Our proof for alternating groups and groups of Lie type in odd characteristic is short, using results in [1, 5, 13, and sporadic groups are handled computationally. This leaves the groups $G(q)$ of Lie type in characteristic 2. Using [9] and other tools, we reduce to consideration of classical groups with $q=2$ or 4 . The proof for these groups occupies most of the paper and uses a similar approach to that in [19, involving character theory, induction on the dimension, and computation to establish base cases.

Although our main focus is on squares, our methods also give rise to the following result on higher powers.

Theorem 2. Let $p>7$ be a prime. Then every element of every finite non-abelian simple group $G$ is a product of two $p^{\text {th }}$-powers. In other words, if $w(x, y)=x^{p} y^{p}$, then $w(G)=G$.

Our proof of this result is short and does not employ detailed character analysis or induction. In fact it works for all finite non-abelian groups $G$ and all primes $p>2$, unless $p=3,5,7$ and $G$ is a Lie type group over $\mathbb{F}_{p}$. Observe that, by the general result of [18, for any two positive integers $m, n$, every element of a sufficiently large finite simple group is a product of an $m^{\text {th }}$-power and an $n^{\text {th }}$-power. After we completed the proofs of Theorems 1 and 2 we learned that the surjectivity of the word $x^{p} y^{p}$ (where $p$ is any prime) on all finite non-abelian simple groups was proved at the same time by Guralnick and Malle using different methods (see 10. for this and related results).

\section{Preliminaries}

We begin with a couple of trivial observations.

Lemma 2.1. Let $G$ be a finite group.

(i) If $x \in G$ is an element of odd order, then $x$ is a square.

(ii) Suppose the number of squares in $G$ is greater than $\frac{1}{2}|G|$. Then every element of $G$ is a product of two squares. 
Lemma 2.2. Let $G$ be a finite group. For $g \in G$, the number of pairs $(x, y) \in G \times G$ such that $g=x^{2} y^{2}$ is

$$
|G| \cdot \sum_{\chi \in \operatorname{Irr}(G), \chi \text { real }} \frac{\chi(g)}{\chi(1)}
$$

Proof. This is a special case of [14, Satz 1].

Proposition 2.3. (i) Theorem 1 holds for alternating groups.

(ii) Theorem 1 holds for simple groups of Lie type over fields of odd characteristic.

(iii) Theorem 1 holds for sporadic simple groups.

Proof. (i) Let $G=\mathrm{A}_{n}$. If $n$ is odd (resp. even), then every element of $G$ is a product of two $n$-cycles (resp. $(n-1)$-cycles), by 13 and 1 . Hence every element is a product of two elements of odd order, giving the conclusion by Lemma 2.1(i).

(ii) Let $G=G(q)$ be a simple group of Lie type over a field $\mathbb{F}_{q}$ with $q$ odd. By a result of Ellers and Gordeev [5, Theorem 3, Corollary], every element of $G$ is a product of two unipotent elements. Since these have odd order, the conclusion follows.

(iii) This follows by a routine check of the character tables of the sporadic groups (available in [7]), using Lemma 2.2.

It follows from the proposition that the only remaining groups to handle are simple groups of Lie type over fields $\mathbb{F}_{q}$ with $q=2^{k}$.

Lemma 2.4. Let $G=G(q), q=2^{k}$. The proportion of elements of $G$ that are of odd order is greater than $\frac{1}{2}$ unless one of the following holds:

(i) $q=2$;

(ii) $G=S p_{2 n}$ (4) or $\Omega_{2 n}^{ \pm}(4)$.

Proof. We use the results of [9, which give estimates for the proportion $s(G)$ of elements of even order in $G$. Theorem 1.1 of $[9$ says that

$$
s(G)<\frac{3}{q-1}+\frac{2}{(q-1)^{2}} .
$$

For $q \geq 8$ this is less than $\frac{1}{2}$, giving the result.

Assume now that $q=4$. If $G=L_{n}(4)$, the proof of [9, 2.3] gives $s(G)<$ $\frac{1}{q-1}+\frac{1}{(q-1)^{2}}<\frac{1}{2}$. If $G=U_{n}(4)$, then counting a little more carefully in the proof of [9, 2.3] also gives $s(G)<\frac{1}{2}$. The same holds for exceptional groups over $\mathbb{F}_{4}$ apart from $G_{2}(4)$ and $F_{4}(4)$, by $[9,3.1]$. For the latter groups, the precise numbers of semisimple elements are listed in [21], and the proportion of them is more than $\frac{1}{2}$.

Lemma 2.5. The conclusion of Theorem 1 holds for the following groups:

(i) $S L_{n}(2)(3 \leq n \leq 6)$;

(ii) $S U_{n}(2)(4 \leq n \leq 9)$;

(iii) $S p_{2 n}(2)(3 \leq n \leq 6)$;

(iv) $S p_{2 n}(4)(n=2,3)$;

(v) $\Omega_{2 n}^{ \pm}(2)(4 \leq n \leq 6)$;

(vi) $\Omega_{8}^{ \pm}(4)$;

(vii) ${ }^{3} D_{4}(2),{ }^{2} F_{4}(2)^{\prime}, F_{4}(2), E_{6}^{ \pm}(2), E_{7}(2)$. 
Proof. For all of these groups except $E_{7}(2)$, we applied Lemma 2.2 to the character table. Some of the tables are available in the Character Table Library of GAP [7; the remainder were constructed directly using the Magma [2] implementation of the algorithm of Unger [27. For $E_{7}(2)$ one finds using [21] that the proportion of semisimple elements in the group is just over $\frac{1}{2}$.

\section{Completion of the proof}

It remains to prove Theorem 1 for the following groups:

$$
\begin{aligned}
& S L_{n}(2)(n \geq 7), \\
& S U_{n}(2)(n \geq 10), \\
& S p_{2 n}(2)(n \geq 7), S p_{2 n}(4)(n \geq 4), \\
& \Omega_{2 n}^{ \pm}(2)(n \geq 7), \Omega_{2 n}^{ \pm}(4)(n \geq 5), \\
& E_{8}(2) .
\end{aligned}
$$

For these groups, the proof follows closely that given in [19]: there we proved that certain key elements $g \in G$ are commutators by using the character theoretic criterion that $g$ is a commutator if $\sum_{\chi \in \operatorname{Irr}(G)} \frac{\chi(g)}{\chi(1)} \neq 0$. In many cases we established this by simply showing that

$$
\sum_{1_{G} \neq \chi \in \operatorname{Irr}(G)} \frac{|\chi(g)|}{\chi(1)}<1 .
$$

Of course (1) is sufficient to prove that the sum in Lemma 2.2 is non-zero and hence that $g$ is equal to $x^{2} y^{2}$ for some $x, y \in G$.

Before proceeding, we eliminate the one exceptional group in the above list.

Lemma 3.1. Every element of $E_{8}(2)$ is a product of two squares.

Proof. The proof of the Ore conjecture for $G=E_{8}(2)$ in [19, $\left.\S 7\right]$ was achieved by establishing that for every $g \in G$, either (1) holds or $g$ lies in a subsystem subgroup of $G$, which is a central product of perfect groups of Lie type of rank at most 4 over $\mathbb{F}_{2}$. By Lemma 2.5, we know that Theorem 1 holds for such subgroups. Hence it holds for $G$.

3.1. Some generic cases. It remains to prove the theorem for the classical groups over $\mathbb{F}_{2}$ and $\mathbb{F}_{4}$ listed above. As in [19] we study a key subset of elements of these groups, defined as follows.

Definition. Let $G=C l_{n}(q)=S L_{n}(q), S U_{n}(q), S p_{n}(q)$ or $\Omega_{n}(q)$. An element $x \in$ $G$ is breakable if it lies in a natural proper subgroup $C l_{r}(q) \times C l_{n-r}(q)$ (stabilizing a non-degenerate $r$-space if $\left.G \neq S L_{n}(q)\right)$ and one of the following holds:

(1) both factors $C l_{r}(q)$ and $C l_{n-r}(q)$ are perfect groups;

(2) $C l_{r}(q)$ is perfect, and the projection of $x$ to $C l_{n-r}(q)$ is a product of two squares in $C l_{n-r}(q)$.

Otherwise, $x$ is unbreakable.

A simple induction argument shows that Theorem 1 for $G=C l_{n}(q)$ follows immediately if we prove that every unbreakable element in $G$ is a product of two squares. Indeed, let $x \in G$ and suppose that $x$ is breakable, so $x=\left(x_{1}, x_{2}\right) \in$ $C l_{r}(q) \times C l_{n-r}(q)$ satisfies (1) or (2) in the above definition. In either case, by induction $x_{1}, x_{2}$ are products of two squares in $C l_{r}(q), C l_{n-r}(q)$, respectively, say $x_{i}=y_{i}^{2} z_{i}^{2}$ for $i=1,2$; then $x=\left(y_{1}, y_{2}\right)^{2}\left(z_{1}, z_{2}\right)^{2}$. 
Lemma 3.2. Theorem 1 holds for $G=S p_{2 n}(2)(n \geq 7), \Omega_{2 n}^{ \pm}(2)(n \geq 7)$, or $\Omega_{2 n}^{ \pm}(4)(n \geq 5)$.

Proof. This follows almost immediately from the proofs of the Ore conjecture for these groups in [19, as follows. First consider $G=S p_{2 n}(2)$. By [11, 6.2], there is a set $\mathcal{W}$ of five Weil characters such that every non-trivial irreducible character of $G$ not in $\mathcal{W}$ has degree at least $\left(2^{2 n}-1\right)\left(2^{n-1}-1\right)\left(2^{n-1}-4\right) / 30$. Moreover, the characters in $\mathcal{W}$ have distinct degrees which are all less than the indicated bound (see [11, §3]), so in particular they are all real. For $x \in G$ let

$$
F_{1}(x)=\sum_{\chi \in \mathcal{W}} \frac{\chi(x)}{\chi(1)}, \quad F_{2}(x)=\sum_{1_{G} \neq \chi \in \operatorname{Irr}(G) \backslash \mathcal{W}} \frac{|\chi(x)|}{\chi(1)} .
$$

By Lemma 2.2 it is sufficient to show that $\left|F_{1}(x)\right|+F_{2}(x)<1$ for all unbreakable $x \in G$. This is established in Lemmas 4.4-4.7 of [19].

An entirely similar discussion applies when $G$ is an orthogonal group.

The remaining groups $S L_{n}(2), S U_{n}(2), S p_{2 n}(4)$ were not handled in the above way in [19, so they require detailed arguments here.

\subsection{Special linear and symplectic groups.}

Lemma 3.3. Theorem 1 holds for $G=S L_{n}(2)(n \geq 7)$.

Proof. Let $x \in G$ be unbreakable. We claim first that

$$
\left|C_{G}(x)\right| \leq 2^{n} \cdot 3^{2} .
$$

The proof is similar to analogous proofs in [19, for example [19, 4.7]. If $x$ is unipotent and unbreakable, then it has only Jordan blocks of size 2 or at least $n-2$ (size 2 is allowed as $S L_{2}(2)$ is non-perfect). It follows easily that $x=J_{n}$ or $\left(J_{n-2}, J_{2}\right)$, where $J_{i}$ denotes a Jordan block of size $i$. These have centralizers of order $2^{n}$ or $2^{n+2}$ respectively, so (2) holds. If $x$ is not unipotent, write $x=s u$ with $s, u$ commuting semisimple and unipotent elements. As $x$ is unbreakable, one of the following holds:

(i) $C_{G}(s)=G L_{n / k}\left(2^{k}\right)$ for some $k$ dividing $n$, and $u=J_{n / k} \in G L_{n / k}\left(2^{k}\right)$;

(ii) $n=2 m, C_{G}(s)=G L_{m}(4)$ and $u=\left(J_{m-1}, J_{1}\right) \in G L_{m}(4)$;

(iii) $n=2 m+2, C_{G}(s)=G L_{m}(4) \times G L_{1}(4)$ or $G L_{m}(4) \times G L_{2}(2)$ and $u=\left(J_{m}, J_{1}\right)$ or $\left(J_{m}, J_{2}\right)$ respectively.

The centralizer orders $\left|C_{G}(x)\right|$ are given by [20, §2], and the largest is that in case (ii), where $\left|C_{G}(x)\right|=4^{m}\left|G L_{1}(4)\right|^{2}$. Hence (2) is proved.

Next, define $D=\left(2^{n}-1\right)\left(2^{n-1}-4\right) / 3$. By [25, Theorem 3.1], $G$ has exactly one non-trivial irreducible character $\chi_{0}$ of degree less than $D$, and $\chi_{0}$ is the non-trivial constituent of the permutation character of $G$ on 1 -spaces and has degree $2^{n}-2$. We saw above that $\operatorname{dim} C_{V}(x) \leq 2$ (where $V=V_{n}(2)$ ), so

$$
\frac{\left|\chi_{0}(x)\right|}{\chi_{0}(1)} \leq \frac{3}{2^{n}-2} \text {. }
$$

The number $k(G)$ of conjugacy classes of $G$ satisfies $k(G) \leq(2.5) \cdot 2^{n-1}$ by [6, 3.6]. Hence by [19, 2.6],

$$
\sum_{\chi \in \operatorname{Irr}(G), \chi(1) \geq D} \frac{|\chi(x)|}{\chi(1)} \leq \frac{k(G)^{1 / 2}\left|C_{G}(x)\right|^{1 / 2}}{D} \leq \frac{\sqrt{2.5} \cdot 2^{(n-1) / 2} \cdot 2^{n / 2} \cdot 3}{D}<0.2 .
$$


It follows that

$$
\sum_{1_{G} \neq \chi \in \operatorname{Irr}(G)} \frac{|\chi(x)|}{\chi(1)}<\frac{3}{2^{n}-2}+0.2<1,
$$

and so by Lemma 2.2 the conclusion follows.

Lemma 3.4. Theorem 1 holds for $G=S p_{2 n}(4)(n \geq 4)$.

Proof. Let $x \in G$ be unbreakable. As $S p_{2}(4)$ is perfect, $x$ cannot stabilize a proper non-degenerate subspace. It follows that if $x$ is unipotent, then $x=J_{2 n}$ or $J_{n}^{2}$ $(V(2 n)$ or $W(n)$ in the notation of [20, $\S 3])$; and if $x=s u$ is not unipotent, then $C_{G}(s)=G L_{n / k}^{ \pm}\left(4^{k}\right)$ and $u=J_{n / k} \in G L_{n / k}^{ \pm}\left(4^{k}\right)$, for some $k$ dividing $n$. The centralizer orders are given in [20], and the largest is for $x=J_{n}^{2}$, which has centralizer order $4^{2 n-1} \cdot\left|S p_{2}(4)\right|$ if $n$ is even and $4^{2 n} \cdot\left|O_{2}^{ \pm}(4)\right|$ if $n$ is odd. Hence

$$
\left|C_{G}(x)\right|<4^{2 n+2} \text {. }
$$

Now we consider characters of $G$. By [11, 6.2], there is a collection $\mathcal{W}$ of 7 irreducible characters such that every non-trivial irreducible character not in $\mathcal{W}$ has degree at least

$$
D=\frac{\left(4^{2 n}-1\right)\left(4^{n-1}-1\right)\left(4^{n-1}-4^{2}\right)}{2\left(4^{4}-1\right)} .
$$

The 7 characters in $\mathcal{W}$ are labelled $\alpha_{n}, \beta_{n}, \rho_{n}^{1}, \rho_{n}^{2}, \tau_{n}^{1}, \zeta_{n}^{1}, \zeta_{n}^{2}$, and all are real. The values of $\alpha_{n}+\beta_{n}, \rho_{n}^{1}+\rho_{n}^{2}, \tau_{n}^{1}, \zeta_{n}^{1}$, and $\zeta_{n}^{2}$ are described explicitly in [11, $\left.\S 3\right]$, and we see easily as in the proof of [19, Lemma 4.5] that

$$
\left|F_{1}(x)\right|=\left|\sum_{\chi \in \mathcal{W}} \frac{\chi(x)}{\chi(1)}\right|<0.2 .
$$

Also $k(G) \leq(15.2) \cdot 4^{n}$ by $[6,3.13]$, so as in the previous proof

$$
F_{2}(x)=\sum_{\chi \in \operatorname{Irr}(G), \chi(1) \geq D} \frac{|\chi(x)|}{\chi(1)} \leq \frac{\sqrt{15.2} \cdot 2^{n} \cdot 4^{n+1}}{D}<0.2 .
$$

Hence $\left|F_{1}(x)\right|+F_{2}(x)<1$ and the conclusion follows.

3.3. Unitary groups. The proof for $S U_{n}(2)$ with $n \geq 10$ is similar to the previous ones, but we give more detail, as unitary groups were handled by a different method in 19 .

Lemma 3.5. If $x \in G=S U_{n}(2)$ is unbreakable, then $\left|C_{G}(x)\right| \leq 2^{3 n-6} \cdot 3^{4}$. Moreover, if $n=10$, then $\left|C_{G}(x)\right| \leq 2^{15} \cdot 3^{4}$.

Proof. The non-perfect special unitary groups are $S U_{r}(2)$ with $r \leq 3$, so $x$ does not lie in a subgroup $S U_{r}(2) \times S U_{n-r}(2)$ with $r \in\{1,2,3\}$. If $x$ is unipotent, then it is $J_{n},\left(J_{n-2}, J_{2}\right)$ or $\left(J_{n-3}, J_{3}\right)$, all of which have a centralizer order smaller than the bound in the conclusion (see [20, $\S 2]$ ). Otherwise $x=s u$ with $s$ a non-trivial semisimple element, and the largest possible centralizer is achieved when $n$ is a multiple of $3, C_{G}(s)=G \cap G U_{3 m}\left(2^{k}\right)(n=3 m k, k$ odd $)$, and $u=\left(J_{m}^{3}\right) \in G U_{3 m}\left(2^{k}\right)$. Writing $q=2^{k}$ we have

$$
\begin{aligned}
\left|C_{G}(x)\right| \leq\left|C_{G U_{3 m}(q)}(u)\right| & =q^{9 m-9}\left|G U_{3}(q)\right| \\
& =2^{3 n} \cdot \frac{\left(q^{3}+1\right)\left(q^{2}-1\right)(q+1)}{q^{6}} .
\end{aligned}
$$


The right hand side attains a maximum when $k=1$ and $q=2$; it achieves the bound in the conclusion. The stronger bound for $n=10$ was established computationally - it occurs for the element $x=\left(\omega J_{2}, \omega J_{2}, \omega J_{3}, x_{0}\right)$ where $\omega$ is a cube root of 1 in $\mathbb{F}_{4}$ and $x_{0}$ is an element of order 9 in $G U_{3}(2)$.

Consider the natural module $V=\mathbb{F}_{4}^{n}$ for $G:=G U_{n}(2)$. Any $g \in G$ is indecomposable if $V$ cannot be decomposed into a direct sum of pairwise orthogonal $g$-invariant non-zero non-degenerate subspaces. A non-zero non-degenerate subspace $U$ of $V$ is a $\lambda$-block for $g$ if $U$ is $g$-invariant, $\left.g\right|_{U}$ is indecomposable, and $\operatorname{det}\left(\left.g\right|_{U}\right)=\lambda$. A block for $g$ is a $\lambda$-block for some $\lambda \in \mathbb{F}_{4}^{\times}=\left\{1, \omega, \omega^{2}\right\}$.

Lemma 3.6. Assume $g \in S U_{n}(2)$ is unbreakable and $n \geq 10$. Then $V$ is a direct sum of at most 6 blocks.

Proof. Write

$$
V=A_{1} \oplus A_{2} \oplus \ldots \oplus A_{r} \oplus B_{1} \oplus B_{2} \oplus \ldots \oplus B_{s} \oplus C_{1} \oplus C_{2} \oplus \ldots \oplus C_{t}
$$

as a direct sum of $r+s+t$ blocks for $g$, where $A_{i}$ is a 1-block of dimension $a_{i}, B_{j}$ is an $\omega$-block of dimension $b_{j}, C_{k}$ is an $\omega^{2}$-block of dimension $c_{k}, r, s, t \geq 0$, and

$$
1 \leq a_{1} \leq \ldots \leq a_{r}, 1 \leq b_{1} \leq \ldots \leq b_{s}, 1 \leq c_{1} \leq \ldots \leq c_{t} .
$$

Arguing by contradiction, we assume that $r+s+t \geq 7$.

1) First we claim that $V$ cannot be written as an orthogonal sum of three $g$ invariant non-degenerate subspaces $X \oplus Y \oplus Z$ with $\operatorname{dim} X, \operatorname{dim} Y, \operatorname{dim} Z \geq 2$ and $\operatorname{det}\left(\left.g\right|_{X}\right)=\operatorname{det}\left(\left.g\right|_{Y}\right)=\operatorname{det}\left(\left.g\right|_{Z}\right)=1$. Indeed, assume the contrary and (without loss) that $x:=\operatorname{dim} X \leq y:=\operatorname{dim} Y \leq z:=\operatorname{dim} Z$. Then $z \geq 4$ as $n \geq 10$, and $x+y \geq 4$. Now observe that $g \in S U_{z}(2) \times S U_{x+y}(2)$, and so $g$ is breakable.

2 ) Here we show that $\min (s, t) \leq 1$, for, assuming that $s, t \geq 2$, we can define

$$
X:=B_{1} \oplus C_{1}, Y:=B_{2} \oplus C_{2}, Z:=(X \oplus Y)^{\perp} .
$$

Then $\operatorname{dim} X, \operatorname{dim} Y \geq 2$ and $\operatorname{dim} Z \geq r+s+t-4 \geq 3$. Furthermore, the choices of $X, Y, Z$ ensure that $g_{X},\left.g\right|_{Y}$, and $\left.g\right|_{Z}$ all have determinant 1 . Hence $g$ is breakable by 1$)$.

3) Next we consider the case $r \geq 2$. If $s, t \geq 1$, then we can define

$$
X:=A_{1} \oplus A_{2}, Y:=B_{1} \oplus C_{1}, Z:=(X \oplus Y)^{\perp}
$$

and conclude that $g$ is breakable by 1 ). Furthermore, if $s \geq 3$ for instance, then we define

$$
X:=A_{1} \oplus A_{2}, Y:=B_{1} \oplus B_{2} \oplus B_{3}, Z:=(X \oplus Y)^{\perp}
$$

and again conclude that $g$ is breakable by 1$)$. So we must have that $\min (s, t)=0$ and $\max (s, t) \leq 2$. Replacing $g$ by $g^{-1}$ if necessary, we may assume that $t=0$ and $s \leq 2$. In this case, $1=\operatorname{det}(g)=\omega^{s}$; hence $s=0$ and $r \geq 7$. Now if $d:=a_{1}+a_{2}+a_{3} \geq 4$, then

$$
g \in S U\left(A_{1} \oplus A_{2} \oplus A_{3}\right) \times S U\left(\left(A_{1} \oplus A_{2} \oplus A_{3}\right)^{\perp}\right)=S U_{d}(2) \times S U_{n-d}(2)
$$

with $n-d \geq 4$, and so $g$ is breakable. So $a_{1}=a_{2}=a_{3}=1$. If in addition $3+a_{4} \leq n-4$, then

$$
g \in S U\left(A_{1} \oplus \ldots \oplus A_{4}\right) \times S U\left(\left(A_{1} \oplus \ldots \oplus A_{4}\right)^{\perp}\right)=S U_{3+a_{4}}(2) \times S U_{n-3-a_{4}}(2)
$$

and again $g$ is breakable. Thus $3+a_{4} \geq n-3$, and so $a_{4} \geq n-6 \geq 4$. This is impossible, since $n-3-a_{4}>a_{5}+a_{6} \geq 2 a_{4} \geq 8$. 
4) We have shown that $r \leq 1$. By 2) we may assume $t \leq 1$, whence $s=n-r-t \geq$ 5 . Since $1=\operatorname{det}(g)=\omega^{s+2 t}$, we must have $3 \mid(s-t)$, and so $s \geq 6$. Defining

$$
X:=B_{1} \oplus B_{2} \oplus B_{3}, Y:=B_{4} \oplus B_{5} \oplus B_{6}, Z:=(X \oplus Y)^{\perp},
$$

by 1 ) we see that the unbreakability of $g$ implies that $\operatorname{dim} Z \leq 1$. However, $\operatorname{dim} Z \geq$ $(r+s+t)-6$, so we must have that $r+s+t=7$ and either $r=t=0$ and $s=7$ or $r+t=1$ and $s=6$. The former is impossible as $3 \mid(s-t)$. Similarly, in the latter case $t=0$ and $r=1$, and moreover $Z=A_{1}$ has dimension 1 . Recalling that $A_{1}$ is a 1-block, we see $\left.g\right|_{A_{1}}=1$, and so $g \in S U_{n-1}(2)$, again a contradiction.

For $\lambda \in \mathbb{F}_{q^{2}}^{\times}$and $g \in G U_{n}(q)$, define $e(g, \lambda)$ to be the dimension (over $\mathbb{F}_{q^{2}}$ ) of $\operatorname{Ker}\left(g-\lambda \cdot 1_{V}\right)$. If $f \in \mathbb{F}_{q^{2}}[t]$ is a monic irreducible polynomial with a root $\alpha \in \overline{\mathbb{F}}_{q^{2}}$, define $\check{f}$ to be the unique monic irreducible polynomial over $\mathbb{F}_{q^{2}}$ such that $\check{f}\left(\alpha^{-q}\right)=0$.

Corollary 3.7. If $n \geq 10$ and $g \in S U_{n}(2)$ is unbreakable, then

$$
\sum_{\lambda \in \mathbb{F}_{4}^{\times}} e(g, \lambda) \leq 6 .
$$

Proof. Consider an $\alpha$-block $U$ of $g$ for $\alpha \in \mathbb{F}_{4}^{\times}$. Since $\left.g\right|_{U}$ is indecomposable, the semisimple part $s$ of $\left.g\right|_{U}$ must have characteristic polynomial $f(t)^{k}$, where $f \in \mathbb{F}_{4}[t]$ is irreducible and $f=\check{f}$, or $(f(t) \check{f}(t))^{k}$, where $f \in \mathbb{F}_{4}[t]$ is irreducible and $f \neq \check{f}$. In both cases, if $\operatorname{deg}(f) \geq 2$, then $f(\lambda) \neq 0$ for $\lambda \in \mathbb{F}_{4}^{\times}$, and so $\operatorname{dim} \operatorname{Ker}\left(\left.g\right|_{U}-\lambda \cdot 1_{U}\right)=0$. On the other hand, if $\operatorname{deg}(f)=1$, then $f(t)=t-\beta$ for some $\beta \in \mathbb{F}_{4}^{\times}$, whence $f=\check{f}$ (i.e. we are in the former case) and $s=\beta \cdot 1_{U}$. Then the indecomposability of $\left.g\right|_{U}$ implies that the unipotent part of $g$ acts on $U$ as a single Jordan block of size $k$. Thus $\operatorname{dim} \operatorname{Ker}\left(\left.g\right|_{U}-\lambda \cdot 1_{U}\right)$ equals 1 if $\lambda=\beta$ and 0 otherwise. Now the statement follows immediately from Lemma 3.6 .

Lemma 3.8. Let $p$ be a prime, $q$ a power of $p$, and let $s \in G:=G U_{n}(q)$ be a semisimple element such that $s$ and $\alpha$ s are conjugate, where $1=\alpha^{q+1} \neq \alpha \in \mathbb{F}_{q^{2}}^{\times}$. Then

$$
N(s):=\left(G: C_{G}(s)\right)_{p^{\prime}}>q^{n^{2} / 4-2} .
$$

Proof. 1) As usual, we may decompose $V$ into an orthogonal sum $V_{1} \oplus \ldots \oplus V_{m}$ of $s$-invariant non-degenerate subspaces, with the characteristic polynomial of $\left.s\right|_{V_{i}}$ being either $f_{i}(t)^{k}$, where $f_{i} \in \mathbb{F}_{q^{2}}[t]$ is irreducible and $f_{i}=\check{f}_{i}$, or $\left(f_{i}(t) \check{f}_{i}(t)\right)^{k}$, where $f_{i} \in \mathbb{F}_{q^{2}}[t]$ is irreducible and $f_{i} \neq \check{f}_{i}$, and moreover $f_{i} \neq f_{j}, \check{f}_{j}$ whenever $i \neq j$. Since $s$ and $\alpha s$ are conjugate, the map $J: x \mapsto \alpha x$ on $\overline{\mathbb{F}}_{q}$ preserves the set of all eigenvalues of $s$. As $\alpha=\alpha^{q^{2}}, J$ induces an action on the set of irreducible factors $f_{i}$ of the characteristic polynomial of $s$. Moreover, since $\alpha=\alpha^{-q}$, this action of $J$ commutes with the map $f_{i} \mapsto \check{f}_{i}$. Thus $J$ induces an action (which we also denote by $J$ ) on the set of summands $V_{1}, \ldots, V_{m}$, with say $l$ orbits. We consider the decomposition

$$
V=W_{1} \oplus W_{2} \oplus \ldots \oplus W_{l},
$$

where each $W_{j}$ is the sum of all $V_{i}$ belonging to one orbit of $J$.

2) Observe that, by our construction, $s_{j}:=\left.s\right|_{W_{j}}$ and $\alpha s_{j}$ are conjugate in $G U\left(W_{j}\right)$. Indeed, if $x s x^{-1}=\alpha s$ for some $x \in G U(V)$, then $\left.s\right|_{x\left(W_{j}\right)}$ has characteristic polynomial equal to the image under $J$ of the characteristic polynomial 
of $\left.s\right|_{W_{j}}$. It follows that $x\left(W_{j}\right)=W_{j}$ and $\left.x\right|_{W_{j}}$ conjugates $s_{j}$ to $\alpha s_{j}$. In particular, $\operatorname{dim} W_{j}>1$ as $\alpha \neq 1$. Furthermore, $C_{G}(s)$ preserves each $W_{j}$ since it fixes each $V_{i}$.

3) The desired bound is obvious when $n \leq 5$. We proceed by induction on $n \geq 6$. First we consider the case $l \geq 2$. The observations in 2) allow us to apply the induction hypothesis to $s_{1}=\left.s\right|_{W_{1}}$ and $s_{1}^{\prime}:=\left.s\right|_{W_{1}^{\prime}}$ with $W_{1}^{\prime}:=\left(W_{1}\right)^{\perp}$. Hence

$$
\begin{aligned}
& N\left(s_{1}\right)=\left(G U\left(W_{1}\right): C_{G U\left(W_{1}\right)}\left(s_{1}\right)\right)_{p^{\prime}}>q^{a^{2} / 4-2}, \\
& N\left(s_{1}^{\prime}\right)=\left(G U\left(W_{1}^{\prime}\right): C_{G U\left(W_{1}^{\prime}\right)}\left(s_{1}^{\prime}\right)\right)_{p^{\prime}}>q^{b^{2} / 4-2},
\end{aligned}
$$

where $a:=\operatorname{dim} W_{1} \geq 2$ and $b:=\operatorname{dim} W_{1}^{\prime} \geq 2$. Observe that

$$
q^{k(k+1) / 2}<f(q, k):=\prod_{i=1}^{k}\left(q^{i}-(-1)^{i}\right) \leq \frac{q+1}{q} \cdot q^{k(k+1) / 2}
$$

for every $k \geq 1$. It follows that

$$
\frac{|G U(V)|_{p^{\prime}}}{\left|\left(G U\left(W_{1}\right) \times G U\left(W_{1}^{\prime}\right)\right)\right|_{p^{\prime}}}=\frac{f(q, n)}{f(q, a) f(q, b)}>\frac{q^{\frac{n(n+1)}{2}}}{\left(\frac{q+1}{q}\right)^{2} \cdot q^{\frac{a(a+1)+b(b+1)}{2}}}>q^{a b-2} .
$$

Since $n=a+b \geq 6$ and $a, b \geq 2$, we have $a b \geq 8$. Consequently,

$$
N(s)>N\left(s_{1}\right) N\left(s_{1}^{\prime}\right) \cdot \frac{f(q, n)}{f(q, a) f(q, b)}>q^{\frac{a^{2}+b^{2}}{4}-4} \cdot q^{a b-2} \geq q^{\frac{n^{2}}{4}-2},
$$

as stated.

4) Now we may assume that $l=1$. First we consider the case where $f_{i}=\check{f}_{i}$. The characteristic polynomial of $s$ is $\left(f_{1} f_{2} \ldots f_{m}\right)^{k}$, and $\operatorname{deg} f_{i}=r$ is odd. Then $C_{G}(s) \cong G U_{k}\left(q^{r}\right)^{m}$ and $n=k r m$. Applying (3) we get

$$
N(s)=\frac{f(q, n)}{f\left(q^{r}, k\right)^{m}}>\frac{q^{\frac{n(n+1)}{2}}}{\left(\frac{q^{r}+1}{q^{r}}\right)^{m} \cdot q^{m r \frac{k(k+1)}{2}}}>q^{\frac{n^{2}}{4}+\frac{n}{4}(m k r-2 k)-\frac{3 m}{5}}
$$

(since $\left.\left(q^{r}+1\right) / q^{r} \leq 3 / 2<q^{3 / 5}\right)$. If $m r \geq 3$ and $n \geq 8$, then $m k r-2 k \geq n / 3$ and $n^{2} / 12>3 m / 5$, yielding $N(s)>q^{n^{2} / 4}$. The same holds if $m r \geq 3$ and $n=6,7$. Assume $m r \leq 2$. Since $r$ is odd, $r=1$. If in addition $m=1$, then $s$ is scalar, and so $s$ and $\alpha s$ cannot be conjugate. Thus $m \geq 2$, and so $N(s)>q^{n^{2} / 4-2}$.

Finally we consider the case where $f_{i} \neq \check{f}_{i}$. The characteristic polynomial of $s$ is $\left(f_{1} f_{2} \ldots f_{m} \check{f}_{1} \check{f}_{2} \ldots \check{f}_{m}\right)^{k}$, and $\operatorname{deg} f_{i}=r \geq 1$. Then $C_{G}(s) \cong G L_{k}\left(q^{2 r}\right)^{m}$ and $n=2 \mathrm{krm}$. Applying (3), we get

$$
N(s)>\frac{f(q, n)}{q^{r m k(k+1)}}>\frac{q^{\frac{n(n+1)}{2}}}{q^{m r k(k+1)}}>q^{\frac{n^{2}}{2}-\frac{n}{2} k} \geq q^{\frac{n^{2}}{4}},
$$

and so we are done with the inductive step.

Now we prove the following theorem, which is of independent interest:

Theorem 3.9. Let $q$ be a power of a prime $p$ and let $\ell=0$ or a prime coprime to $\operatorname{gcd}(n, q+1)$. Assume $V$ is an $\ell$-modular absolutely irreducible representation of $G U_{n}(q)$ which is reducible on restriction to $S U_{n}(q)$. Then

$$
\operatorname{dim} V>q^{\frac{n^{2}}{4}-2} \text {. }
$$


Proof. 1) Let $G:=G U_{n}(q), S:=S U_{n}(q), Z:=Z(G)$. Consider the subgroups $A, B$ of $G$ which contain $S$ and such that $A / S=O_{\ell}(G / S)$ and $B / S=O_{\ell^{\prime}}(G / S)$. Since $(G: Z S)=\operatorname{gcd}(n, q+1)$ is coprime to $\ell$, we have $A \leq Z S$. For $X \triangleleft G$, let $\kappa_{X}^{G}(V)$ denote the total number of irreducible constituents of the $X$-module $\left.V\right|_{X}$. Similarly, we choose $U$ to be an irreducible constituent of the $A$-module $\left.V\right|_{A}$, and let $\kappa_{Y}^{A}(U)$ denote the total number of irreducible constituents of the $Y$-module $\left.U\right|_{Y}$ for $Y \triangleleft A$. Since $A \leq Z S$, every $S$-irreducible constituent $W$ of $\left.U\right|_{S}$ is $A$-invariant. But $A / S$ is cyclic; hence $W$ extends to $A$. In other words, $\kappa_{S}^{A}(U)=1$. By our assumptions, $\kappa_{S}^{G}(V)>1$, and by [15, Lemma 3.3],

$$
\kappa_{S}^{G}(V)=\kappa_{A}^{G}(V) \cdot \kappa_{S}^{A}(U) .
$$

Hence $\kappa_{A}^{G}(V)>1$.

Recall that $G / A$ is a cyclic $\ell^{\prime}$-group. The latter inequality implies by [15, Lemma 3.2] that there is some non-trivial irreducible $\ell$-modular representation $L$ of $G$ which is trivial on $A$ such that $V \simeq V \otimes L$.

2) Observe that the dual group $G^{*}$ of $G=G U_{n}(q)$ can be identified with $G$. Hence, $\operatorname{Irr}(G)$ is the disjoint union of the rational series $\mathcal{E}(G,(x))$, where $(x)$ runs over the set of conjugacy classes $(x)$ of semisimple elements $x \in G$; cf. [4, 22]. Furthermore, according to the main result of $[3, \operatorname{Irr}(G)$ can be partitioned into the disjoint union of $\mathcal{E}_{\ell}(G,(y))$, where each $\mathcal{E}_{\ell}(G,(y))$, labelled by the conjugacy class (y) of semisimple $\ell^{\prime}$-elements $y \in G$ and defined by

$$
\mathcal{E}_{\ell}(G,(y))=\bigcup_{t \in C_{G}(y), t \text { is an } \ell \text {-element }} \mathcal{E}(G,(y t)),
$$

is a union of $\ell$-blocks.

Assume that $V$ belongs to the union $\mathcal{E}_{\ell}(G,(s))$ of blocks labelled by the conjugacy class of a semisimple $\ell^{\prime}$-element $s \in G$. Since $S$ acts trivially on $L$ but $L$ is nontrivial, we can also find a non-trivial $\ell^{\prime}$-element $z \in Z$ such that the Brauer character of $L$ is just the restriction to $\ell^{\prime}$-elements in $G$ of the semisimple character $\chi_{z}$ labelled by $z$. According to [4, Proposition 13.30] and its proof, the tensor product with $\chi_{z}$ defines a bijection between the series $\mathcal{E}(G,(x))$ and $\mathcal{E}(G,(x z))$, hence also between the unions of blocks $\mathcal{E}_{\ell}(G,(s))$ and $\mathcal{E}_{\ell}(G,(s z))$. Since $V \simeq V \otimes L$, we conclude that $(s)=(s z)$, i.e. $s$ and $s z$ are conjugate in $G$. By Lemma 3.8 $N(s)=(G$ : $\left.C_{G}(s)\right)_{p^{\prime}}>q^{n^{2} / 4-2}$. Finally, by [12, Proposition 1], $\operatorname{dim} V$ is divisible by $N(s)$, whence the statement follows.

Lemma 3.10. Let $S=S U_{n}(2)$ with $n \geq 10$ and let

$$
D= \begin{cases}\frac{\left(2^{n}-1\right)\left(2^{n-1}+1\right)\left(2^{n-2}-1\right)}{3^{4}}, & n \text { even }, \\ \frac{\left(2^{n}+1\right)\left(2^{n-1}-1\right)\left(2^{n-2}-2^{3}\right)}{3^{4}}, & n \text { odd } .\end{cases}
$$

If $1_{S} \neq \chi \in \operatorname{Irr}(S)$ and $\chi(1)<D$, then $\chi$ is either one of three Weil characters or one of the characters $D_{\alpha}^{\circ}$ defined in [19, Proposition 6.3].

Proof. Let $G=G U_{n}(2)$ and let $\theta \in \operatorname{Irr}(G)$ be such that $\chi$ is an irreducible constituent of $\left.\theta\right|_{S}$. Clearly, $\theta(1) \leq 3 \chi(1)<3 D<2^{n^{2} / 4-2}$. It then follows by Theorem 3.9 that $\left.\theta\right|_{S}$ is irreducible, whence $\chi=\left.\theta\right|_{S}$. In particular, $\theta(1)=\chi(1)<D$. Now the statement follows from [19, Proposition 6.6]. 
Lemma 3.11. If $g \in S=S U_{n}(2)$ is unbreakable, $n \geq 10$, and $D$ is as in the previous lemma, then

$$
F_{1}(g)=\sum_{1<\chi(1)<D, \chi \text { real }} \frac{|\chi(g)|}{\chi(1)}< \begin{cases}0.09, & \text { if } n \geq 11, \\ 0.28, & \text { if } n=10 .\end{cases}
$$

Proof. 1) It is well known that among the three Weil characters $\zeta_{n, 2}^{i}$ of $S$, only the unipotent character $\zeta:=\zeta_{n, 2}^{0}$ is real. Next we show that the character $D_{\alpha}^{\circ}$ is real if and only if $\alpha \in \operatorname{Irr}\left(G U_{2}(2)\right)$ is real. The characters $D_{\alpha}^{\circ}$ are constructed in [19, §6.1] by embedding a central product $H * S$ in $G U_{2 n}(2)$ for $H:=G U_{2}(2)$ and restricting the reducible Weil character

$$
\varphi(g)=(-2)^{\operatorname{dim}_{F_{4}} \operatorname{Ker}(g-1)}
$$

of $G U_{2 n}(2)$ to $H * S$. In particular,

$$
\left.\varphi\right|_{H * G}=\sum_{\alpha \in \operatorname{Irr}(H)} \alpha \otimes D_{\alpha} .
$$

By [19, Proposition 6.3], $D_{\alpha}^{\circ}=D_{\alpha}-\kappa_{\alpha} \cdot 1_{S}$ with $\kappa_{\alpha} \in\{0,1\}$; in particular, $D_{\alpha}^{\circ}$ is real if and only if $D_{\alpha}$ is real. Since $\varphi$ is real,

$$
\sum_{\alpha \in \operatorname{Irr}(H)} \bar{\alpha} \otimes \bar{D}_{\alpha}=\left.\bar{\varphi}\right|_{H * G}=\left.\varphi\right|_{H * G}=\sum_{\alpha \in \operatorname{Irr}(H)} \bar{\alpha} \otimes D_{\bar{\alpha}}
$$

whence $\bar{D}_{\alpha}=D_{\bar{\alpha}}$. Also by [19, Proposition 6.3], for $\alpha, \beta \in \operatorname{Irr}(H), D_{\alpha}=D_{\beta}$ precisely when $\alpha=\beta$. We conclude that $D_{\alpha}^{\circ}$ is real precisely when $\alpha$ is real.

2) Observe that $H=G U_{2}(2)$ has exactly three real irreducible characters: $\alpha_{1}=$ $1_{H}, \alpha_{2}$ the Steinberg character (of degree 2), and one more, $\alpha_{3}$, of degree 1 (which is $\chi_{q-1}^{(1,2)}$ in the notation of Table III of [19]). Thus the summation in $F_{1}(g)$ involves 4 characters: $\zeta$, and $\chi_{i}=D_{\alpha_{i}}^{\circ}$ for $1 \leq i \leq 3$. First by [26, Lemma 4.1],

$$
\zeta(g)=\frac{(-1)^{n}}{3} \sum_{\lambda \in \mathbb{F}_{4}^{\times}}(-2)^{e(g, \lambda)} .
$$

By Corollary 3.7. $\sum_{\lambda \in \mathbb{F}_{4}^{\times}} e(g, \lambda) \leq 6$. It follows that $|\zeta(g)| \leq\left(2^{6}+2\right) / 3=22$. Next,

$$
D_{\alpha}(g)=\frac{1}{|H|} \sum_{x \in H} \overline{\alpha(x)} \varphi(x g)
$$

for $\alpha \in \operatorname{Irr}(H)$. The computations in the proof of [19, Proposition 6.9] and Corollary 3.7 show that, for every $x \in H,|\varphi(x g)| \leq 2^{12}$. Since $|\alpha(x)| \leq \alpha(1)$, it follows that $\left|D_{\alpha}(g)\right| \leq 2^{12} \alpha(1)$, and so $\left|D_{\alpha}^{\circ}(g)\right| \leq 2^{12} \alpha(1)+1$. Now for $i=1,3$ we have $\alpha_{i}(1)=1$ and $\chi_{i}(1) \geq\left(2^{n}-2\right)\left(2^{n-1}-4\right) / 9$, whereas for $i=2$ we have $\alpha_{i}(1)=2$ and $\chi_{i}(1)>2\left(2^{n}-2\right)\left(2^{n-1}-4\right) / 9$; cf. [19, Table III]. Also, $\zeta(1) \geq\left(2^{n}-2\right) / 3$. It follows that

$$
F_{1}(g)<\frac{66}{2^{n}-2}+2 \cdot \frac{2^{12}+1}{\left(2^{n}-2\right)\left(2^{n-1}-4\right) / 9}+\frac{2^{13}+1}{2\left(2^{n}-2\right)\left(2^{n-1}-4\right) / 9},
$$

which is less than 0.09 if $n \geq 11$ and 0.28 if $n=10$.

Proposition 3.12. Theorem 1 holds for $G=S U_{n}(2)$ with $n \geq 10$. 
Proof. It suffices to show that every unbreakable $g \in G$ is a product of two squares. We have $k(G)<(8.26) \cdot 2^{n-1}$ by [6, 3.10], so in the usual fashion we see that

$$
F_{2}(g)=\sum_{\chi(1) \geq D} \frac{|\chi(g)|}{\chi(1)} \leq \frac{\sqrt{8.26} \cdot 2^{(n-1) / 2} \cdot 2^{(3 n-6) / 2} \cdot 3^{2}}{D},
$$

where $D$ is as defined in Lemma 3.10, For $n \geq 11$ this yields $F_{2}(g)<0.74$. For $n=10$ we use the stronger bound for $\left|C_{G}(x)\right|$ in Lemma 3.5 to obtain $F_{2}(x) \leq$ $\sqrt{8.26} \cdot 2^{9 / 2} \cdot 2^{15 / 2} \cdot 3^{2} / D$, which is less than 0.07 . On the other hand, $F_{1}(g)<0.09$ for $n \geq 11$ and $F_{1}(g)<0.28$ for $n=10$ by Lemma 3.11. Thus $F_{1}(g)+F_{2}(g)<0.83$ for all unbreakable $g \in G$, and so the statement follows.

This completes the proof of Theorem 1.

\section{Higher POWERS}

We now prove Theorem 2, The arguments are similar to the preliminary part of the proof of Theorem 1

Fix an odd prime $p$. Let $\sigma \in \mathrm{A}_{n}$ for $n \geq 5$ be a longest cycle whose length $m$ is prime to $p$. Then $n-2 \leq m \leq n, \sigma$ is a $p^{\text {th }}$-power, and for $n \geq 8$ every element of $\mathrm{A}_{n}$ is a product of two conjugates of $\sigma$ by [1] and [13. This shows that $x^{p} y^{p}$ is surjective on $\mathrm{A}_{n}$ for $n \geq 8$. The cases $n=5,6,7$ are easily checked directly.

The result for sporadic groups is established computationally, using the character tables available in [7] and the formula

$$
\left|\left\{(x, y) \in G \times G \mid g=x^{p} y^{p}\right\}\right|=|G| \cdot\left(\sum_{\chi \in \operatorname{Irr}(G)} \nu_{p}(\chi)^{2} \cdot \frac{\chi(g)}{\chi(1)}\right)
$$

for $g \in G$, where $\nu_{p}(\chi):=\left(\sum_{h \in G} \chi\left(h^{p}\right)\right) /|G| ;$ cf. [14, Satz 1]. Note that only primes $p$ dividing $|G|$ need to be checked.

Let $G=G(q)$, a simple group of Lie type over $\mathbb{F}_{q}$. If $q$ is prime to $p$, then unipotent elements in $G$ are $p^{\text {th }}$-powers; hence the result follows from [5].

Suppose now that $q=p^{k}$. Arguing as in the proof of Lemma 2.4, we see that $x^{p} y^{p}$ is surjective on $G=G(q)$ if $q \geq 8$. Since $q \geq p$ the result follows for $p>7$ as required. In fact, our method also yields the surjectivity of the word $x^{p^{k}} y^{p^{k}}$ on every non-abelian finite simple group for $p>7$.

\section{REFERENCES}

1. E. Bertram, Even permutations as a product of two conjugate cycles, J. Comb. Theory Ser. A 12 (1972), 368-380. MR0297853(45:6905)

2. W. Bosma, J. Cannon and C. Playoust, The Magma algebra system. I: The user language, J. Symbolic Comput. 24 (1997), 235-265. MR1484478

3. M. Broué and J. Michel, Blocs et séries de Lusztig dans un groupe réductif fini, J. Reine Angew. Math. 395 (1989), 56-67. MR983059 (90b:20037)

4. F. Digne and J. Michel, Representations of Finite Groups of Lie Type, London Mathematical Society Student Texts, 21, Cambridge University Press, 1991. MR.1118841 (92g:20063)

5. E.W. Ellers and N. Gordeev, On the conjectures of J. Thompson and O. Ore, Trans. Amer. Math. Soc. 350 (1998), 3657-3671. MR1422600 (98k:20022)

6. J. Fulman and R. M. Guralnick, Bounds on the number and sizes of conjugacy classes in finite Chevalley groups, Trans. Amer. Math. Soc. (to appear).

7. The GAP Group, GAP - Groups, Algorithms, and Programming, Version 4.4.12, 2008, http://www.gap-system.org. 
8. S. Garion and A. Shalev, Commutator maps, measure preservation, and T-systems, Trans. Amer. Math. Soc. 361 (2009), 4631-4651. MR2506422 (2010f:20019)

9. R.M. Guralnick and F. Lübeck, On $p$-singular elements in Chevalley groups in characteristic p, in: Groups and computation, III (Columbus, OH, 1999), pp. 169-182, Ohio State Univ. Math. Res. Inst. Publ., 8, de Gruyter, Berlin, 2001. MR.1829478 (2002d:20074)

10. R.M. Guralnick and G. Malle, Products of conjugacy classes and fixed point spaces, arXiv:1005.3756 (preprint).

11. R. Guralnick and P.H. Tiep, Cross characteristic representations of even characteristic symplectic groups, Trans. Amer. Math. Soc. 356 (2004), 4969-5023. MR2084408 (2005j:20012)

12. G. Hiss and G. Malle, Low dimensional representations of special unitary groups, J. Algebra 236 (2001), 745-767. MR1813499 (2001m:20019)

13. D. Husemoller, Ramified coverings of Riemann surfaces, Duke Math. J. 29 (1962), 167-174. MR.0136726 (25:188)

14. A. Kerber and B. Wagner, Gleichungen in endlichen Gruppen, Arch. Math. 35 (1980), 252262. MR583596 (81k:20015)

15. A.S. Kleshchev and P.H. Tiep, Representations of finite special linear groups in non-defining characteristic, Adv. Math. 220 (2009), 478-504. MR2466423 (2009j:20022)

16. M. Larsen and A. Shalev, Characters of symmetric groups: Sharp bounds and applications, Invent. Math. 174 (2008), 645-687. MR2453603 (2010g:20022)

17. M. Larsen and A. Shalev, Word maps and Waring type problems, J. Amer. Math. Soc. 22 (2009), 437-466. MR2476780 (2010d:20019)

18. M. Larsen, A. Shalev and P.H. Tiep, The Waring problem for finite simple groups, Annals of Math. (to appear).

19. M.W. Liebeck, E.A. O'Brien, A. Shalev and P.H. Tiep, The Ore Conjecture, J. Europ. Math. Soc. 12 (2010), 939-1008. MR2654085

20. M.W. Liebeck and G.M. Seitz, Unipotent and nilpotent classes in simple algebraic groups and Lie algebras, preprint.

21. Frank Lübeck, Data for Finite Groups of Lie Type and Related Algebraic Groups. www. math.rwth-aachen. de/ Frank. Luebeck/chev.

22. G. Lusztig, Characters of Reductive Groups over a Finite Field, Annals of Math. Studies, 107, Princeton Univ. Press, Princeton, 1984. MR742472 (86j:20038)

23. D. Segal, Words: Notes on verbal width in groups, London Math. Soc. Lecture Note Series, 361, Cambridge University Press, Cambridge, 2009. MR2547644(2011a:20055)

24. A. Shalev, Word maps, conjugacy classes, and a non-commutative Waring-type theorem, Annals of Math. (2) 170 (2009), 1383-1416. MR2600876

25. P.H. Tiep and A. Zalesskii, Minimal characters of the finite classical groups, Comm. Algebra 24 (1996), 2093-2167. MR 1386030 (97f:20018)

26. P.H. Tiep and A.E. Zalesskii, Some characterizations of the Weil representations of the symplectic and unitary groups, J. Algebra 192 (1997), 130-165. MR.1449955 (99d:20074)

27. W.R. Unger, Computing the character table of a finite group, J. Symbolic Comput. 41 (2006), 847-862. MR2246713 (2007b:20023)

Department of Mathematics, Imperial College, Queen's Gate, London SW7 2BZ, UNITED KingDOM

E-mail address: m.liebeck@imperial.ac.uk

Department of Mathematics, University of Auckland, Private Bag 92019, Auckland, NeW ZEALAND

E-mail address: e.obrien@auckland.ac.nz

Institute of Mathematics, Hebrew University, Jerusalem 91904, Israel

E-mail address: shalev@math.huji.ac.il

Department of Mathematics, University of Arizona, 617 N. Santa Rita Avenue, TucSON, ARIZONA 85721

E-mail address: tiep@math.arizona.edu 